\title{
The Gradational Route Planning for Aircraft Stealth Penetration Based on Genetic Algorithm and Sparse A-Star Algorithm
}

\author{
LI Maoquan ${ }^{1}$, ZHANG Yunfei ${ }^{1}$ and LI Shihao ${ }^{1}$ \\ ${ }^{1}$ School of Aeronautic Science and Engineering, Beihang University, Beijing 100191, China
}

\begin{abstract}
It is established for a gradational route planning algorithm which includes two layers. The first layer makes use of genetic algorithm to obtain the global optimal path by its global optimal characteristics. The second layer makes use of $\mathrm{A}^{*}$ algorithm to obtain the local optimal path by its dynamic characteristic. When flying along the global optimal path, locating the new threat and confirming its performance, the aircraft can plan the local optimal path timely by $\mathrm{A}^{*}$ algorithm. It is constructed for the cost function with two goals of the range and the average detection probability, which is used as the goal function for optimal path planning. Two paths that obtained from two optimal methods are merged to construct the optimal route comprehensively considering the threats and range. The simulation result shows that the cost of new optimal route is lower than the original optimal path obtained only by the genetic algorithm.It revealed that our algorithm could obtain an optimal path when a new radar threas occured.
\end{abstract}

\section{Introduction}

Aircraft path planning is to get an optimal or satisfactory flight path to ensure the success of missions, synthetically considering the aircraft arrival time, fuel consumption, threats and other factors[1][2]. In modern warfare, with the increasing performance of air defense systems, aircraft safety penetration is the key to achieving precision strike. So the low altitude penetration path planning is becoming more and more important.

In the artificial intelligence algorithms[3], the genetic algorithm has strong robustness, characterized by flexible and simple implementation, without being limited by the search space. It is a kind of parallel global search algorithm, but it has a long planning time and is not suitable for real-time path planning. $A^{*}$ algorithm due to the introduction of the heuristic function, is able to avoid the blind search[4], which greatly improves the efficiency of the search.

In this paper, a gradational route planning algorithm is established, including the first layer using genetic algorithm to obtain the global optimal path by its global optimal characteristics based on the known threats data, and the second layer using $\mathrm{A}^{*}$ algorithm to obtain the local optimal path by its dynamic characteristic facing the new threat. Combining the global route optimization and dynamic route optimization, this algorithm can play advantages and avoid shortcomings.

\section{Radar model and Radar network integrated detection model}

In this paper the so-called "critical scattering cross-section" method is used to calculate the detection capability of single-step radar[5].

$$
\sigma_{c r\left(\hat{p}, P_{f a}\right)}^{(c+n)}=\sigma_{c r\left(\hat{p}, P_{f a}\right)}^{(n)}+\frac{\sigma_{c}}{F^{4}}\left(\frac{S}{N}\right)_{\min \left(\hat{p}, P_{f a}\right)}
$$

Considering the radar cross section of target, the signal to noise ratio(SNR) is.

$$
\frac{S}{N}=\frac{\sigma}{\sigma_{\operatorname{cr}\left(\hat{p}_{f a}\right)}^{(c+n)}}
$$

According to the relationship of detection probability with SNR for the pulse integration number of a given radar, the detection probability of the radar to the target under certain false alarm probability can be calculated.

The information fusion center of the radar network integrates the information detected by the single radar to obtain the comprehensive detection information[6][7]. Data fusion often uses the Rank $\mathrm{K}$ fusion rule, that is, assuming that there are $\mathrm{n}$ radars, and if there is at least $\mathrm{K}$ radars to determine the target, the radar network determines the target[8]. When $\mathrm{K}=1$, data fusion is called "OR rule". For the distributed radar network, assuming the detection statistics are independent and the detection probability is $P_{d, k}$, then the detection probability of at least one radar found in the radar network is expressed as:

$$
P_{D}=1-\prod_{k=1}^{n}\left(1-P_{d}(k)\right)
$$




\section{Genetic algorithm}

\subsection{Gene code}

In order to discretize the planning area based on simulation step size and referring to the above constraints, the change of course is not more than $45^{\circ}$. Divide the possible courses within each grid into five values[9], as shown in Figure 1.

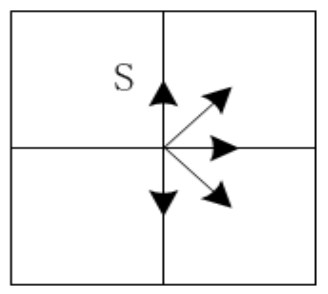

Figure 1. Node's directions

\subsection{Selection operator}

This paper combines the optimum maintaining tactics with the remainder stochastic sampling[4]. The specific process is as follows:

1) Calculate the expected number $N_{i}$ in the next generation for each individual in the population.

$$
N_{i}=M \cdot F_{i} / \sum_{i=1}^{M} F_{i}
$$

$F_{i}$ is the fitness value of the current individual; $M$ is the population size.

2) The integer part of $N_{i}$ is the number of survivors of the individual in the next generation, so that $N$ individuals in the next generation can be determined.

$$
N=\sum_{i=1}^{M} \text { floor }\left(N_{i}\right)
$$

\subsection{Crossover operator}

This paper uses the method of reconstructing a small track near the intersection to ensure that the matching tracks can be crossed successfully, as shown in Figure 2.

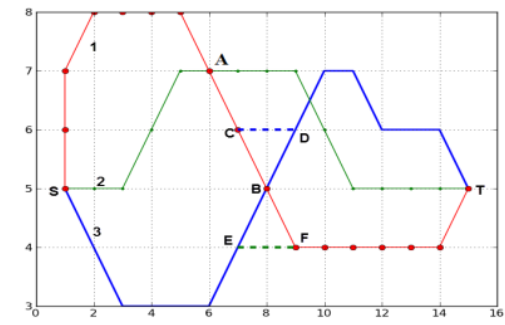

Figure 2. Crossover operator

In the above figure, the two paths 1 and 3, due to not meet the corner of the constraints at the B node which is invalid generally for the intersection, will give up the cross point. The following measures are adopted to complete the two tracks cross. C, D, E, and F are selected near the two track B nodes, and then the track segments (BE segment, DC segment) are truncated.

\subsection{Mutation operator}

In this paper, the mutation operator adopts the method of combining reconstruction operator and disturbance operator. In the mutation, select an operator randomly to operate. The specific operation is as follows:

Reconstruction operator: first select a node as the starting node of the mutation on the track, such as the A node in Figure.3. Then find a track segment (ABT segment) which is different from the original track from the beginning point of the mutation and the target node and satisfies the track constraints, and replace of the original track segment (ADT segment).

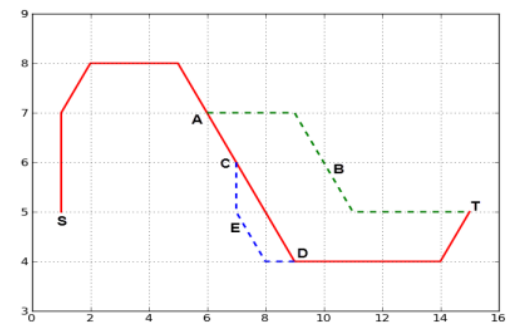

Figure 3. Mutation operator

Perturbation operator: Firstly, a node is randomly selected as the starting node of the disturbance operation, such as the $\mathrm{C}$ node in Figure 3. Then reconstruct a segment (CED segment) to the end node of the mutation (D). If the segment still satisfies the track constraints, the mutation is successful.

\subsection{Cost function of genetic algorithm}

Assuming that the radar network of n-radars has a comprehensive detection probability $P_{C, j}$ of the target at point $j$, the avoidance capability of a route can be evaluated by the average comprehensive detection probability of the route of $\mathrm{m}$-point.

$$
\overline{P_{D}}=\frac{1}{m} \sum_{j=1}^{m} P_{D, j}
$$

The length and the detection probability are commonly not on the same order of magnitude $(0-1)$. Therefore, it needs to be normalized.

$$
L_{k}=\frac{\sum_{j=1}^{n} L_{k, j}-L_{\min }}{L_{\max }-L_{\min }}
$$

Where:

$L_{\text {min }}$ is the shortest range, that is, the straight distance from the starting point to the end point; $L_{\max }$ is the maximum range from the start to the end.

The cost function of the genetic algorithm is finally rewritten as follows:

$$
J=w_{1} L_{k}+w_{2} \overline{P_{D}}
$$




\section{A-Star algorithm}

\subsection{Sparse $A^{*}$ algorithm}

The sparse A* algorithm introduces the constraints of the path planning into the $\mathrm{A} *$ algorithm[9], which can eliminate many sub-nodes that do not satisfy the constraint, and reduce the computational complexity. Since this article specifies that the maximum turning angle within a grid cell should be less than $45^{\circ}$, there are actually at most three sub-nodes that satisfy the constraints, as shown in Figure 4.

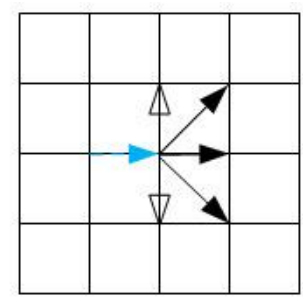

Figure 4. node's directions of sparse A* algorithm

\subsection{Cost function of sparse $A^{*}$ algorithm}

The evaluation function[10] of the $\mathrm{A} *$ algorithm is

$$
f(n)=g(n)+h(n)
$$

where $f(n)$ is the total cost from the start node to the current point, $g(n)$ is the actual cost from the start node to the current node, $h(n)$ as the heuristic function, represents the cost from the current node to the target point.

\section{Gradational path planning}

The above algorithms have their own advantages and disadvantages. The advantage of genetic algorithm is that the path generated is global optimum, but the disadvantage is that the process may not be convergence and the computation time increases with the increase of precision. So it is not suitable as the real-time planning algorithm. A* algorithm has the advantage of fast computation, and the computation time won't be affected by the calculation precision. But the result may be the local optimum. The gradational path planning is an optimization algorithm that combines the advantages of the two algorithms.

The gradational path planning requires the global optimal path is obtained by genetic algorithm based on the known threats and terrain data before flight, and the path is used as the standard path. During the flight, through the threat detection device to detect new threats. When the new threat occurs, the aircraft conduct realtime path planning by $A^{*}$ algorithm, and update the standard path by the new path to avoid new threat.

In Figure 5, the path SMBNE is the optimal path for the known threats by genetic algorithm, and $\mathrm{S}$ and $\mathrm{E}$ are the start point and end point of the path. A new threat $\mathrm{C}$ is detected when the aircraft is flying to point $\mathrm{M}$. In order to avoid the new threat, the aircraft will use the $A^{*}$ algorithm to generate a new path, and then return to the original optimization path after bypassing the new threat to avoid other threats on the battlefield. The idea is that draw a circle with the center $\mathrm{C}$ and the radius $\mathrm{CM}$ and the intersection $\mathrm{M}$ and $\mathrm{N}$ of the optimization path are the start point and the end point of the $\mathrm{A}^{*}$ algorithm. In this way, a track (such as MDN) is found between $\mathrm{M}$ and $\mathrm{N}$ to make the cost value lower than the original track MBN. If the distance of $\mathrm{CM}$ is larger than the distance between $\mathrm{C}$ and the end point $E$, then $E$ is taken as the end of the $A^{*}$ algorithm.

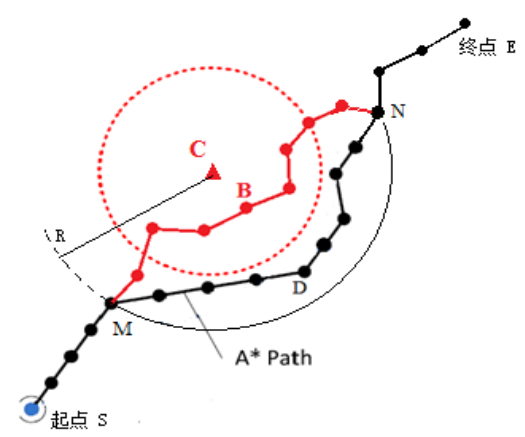

Figure 5. diagram of gradational path

\section{Simulation results and analysis}

\subsection{Battlefield setting}

Assuming the size of the planning area is $400 \mathrm{~km} \times 400 \mathrm{~km}$, and the number of grids is $100 \times 100$, so the size of each grid is $4 \mathrm{~km} \times 4 \mathrm{~km}$.

The ground threat system uses two types of radars as shown in Table.1. And the sites of radars are shown in Table.2.

The aircraft target is spherical target and its $\mathrm{RCS}=$ $5 \mathrm{dBm}^{2}$. The start point is $(0,260,3)$, and the end point is $(400,130,3)$. The range factor $w_{2}=0.6$.

Table 1. Radar performance

\begin{tabular}{ccc}
\hline Performance & AN/ SPS-48 & TRMS \\
\hline Center frequency $(\mathrm{GHz})$ & 3 & 5.6 \\
Polarization mode & $\mathrm{HH}$ & $\mathrm{HH}$ \\
Maximum detection range $(\mathrm{km})$ & 230 & 100 \\
Radar characteristic constants & 8.203 & 0.409 \\
\hline$\left(10^{21} \mathrm{~m}^{2}\right)$ & & \\
\hline
\end{tabular}

Table 2. Coordinates of radar

\begin{tabular}{ccccc}
\hline No & Type & $\mathbf{X} / \mathbf{k m}$ & Y/km & $\mathbf{Z} / \mathbf{k m}$ \\
\hline A & TRMS & 140 & 100 & 0.5 \\
B & TRMS & 160 & 270 & 0.5 \\
C & AN/SPS-48 & 280 & 380 & 0.5 \\
D & TRMS & 300 & 190 & 0.5 \\
\hline
\end{tabular}

6.2 Simulation results of genetic algorithm 
Figure 6 contains the cloud diagram of comprehensive detection probability and the optimized path computed by genetic algorithm in the case of known the distributions of radars $\mathrm{A}, \mathrm{B}$ and $\mathrm{C}$. This path is used as the original path in the dynamic planning.

Figure 7 is the cloud diagram after the new threat $\mathrm{D}$ is detected, which shows a portion of the original path entered the detection area of the radar D.

In contrast, the comprehensive detection probability of the original path after the addition of radar D is significantly improved as shown in Table 3.

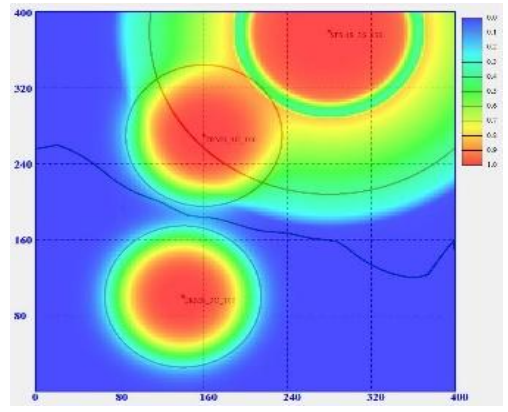

Figure 6. Standard path generated by genetic algorithm

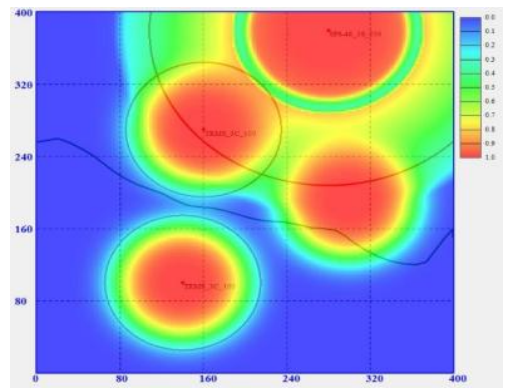

Figure 7. New detection probability distribution

Table 3. The comprehensive detection probability

\begin{tabular}{cc}
\hline Radars & Comprehensive detection probability \\
\hline A,B,C & 0.103 \\
A,B,C,D & 0.716 \\
\hline
\end{tabular}

For the aforementioned radars distribution and the original path, it is assumed that the aircraft finds the new radar D and calculates its threat area before arriving at point $\mathrm{M}$. The dynamic planning simulation is carried out on the aircraft at three positions $\mathrm{M}, \mathrm{N}$ and $\mathrm{Q}$ respectively. (Figure 8.)

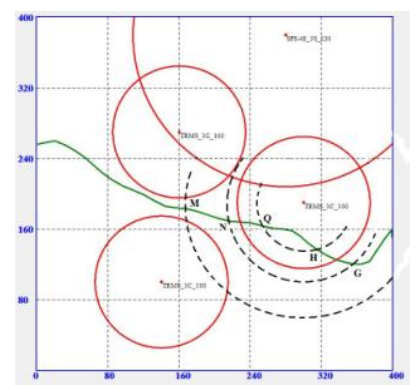

Figure 8. The start and end points of dynamic planning
With the point $\mathrm{M}$ as the start point for dynamic planning, the circle with the radius DM has no second intersection, then select the end of the path as the dynamic path planning end point. The length of the final path is $462 \mathrm{~km}$, the comprehensive detection probability is 0.143 and the cost value is 0.215 . (Figure 9.)

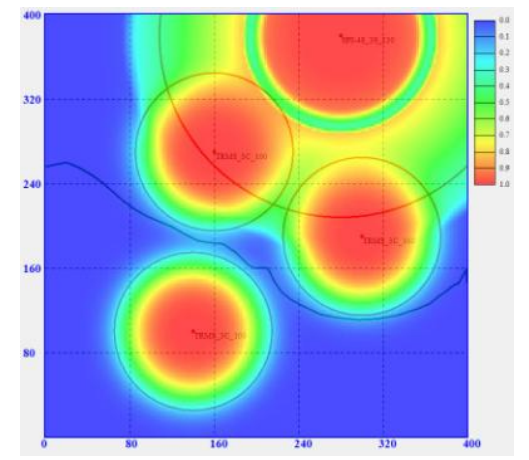

Figure 9. Gradational optimal path with point $\mathrm{M}$

When point $\mathrm{N}$ is the start point for dynamic path planning, the point $\mathrm{G}$ is the end point. The length of the final path is $474 \mathrm{~km}$, the comprehensive detection probability is 0.242 and the cost value is 0.293 . (Figure 10.)

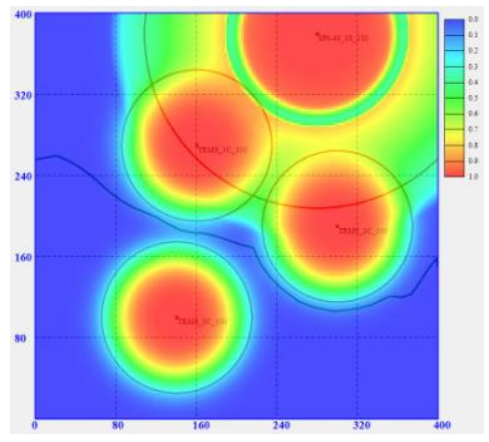

Figure 10. Gradational optimal path with point $\mathrm{N}$

When point $\mathrm{Q}$ is the start point for dynamic path planning, the point $\mathrm{H}$ is the end point. The length of the final path is $472 \mathrm{~km}$, the comprehensive detection probability is 0.593 and the cost value is 0.429 . (Figure 11.)

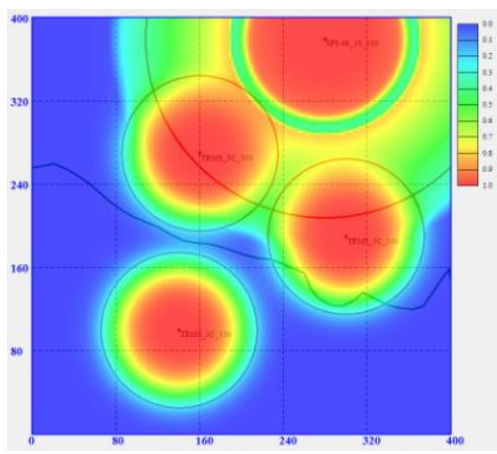

Figure 11. Gradational optimal path with point $\mathrm{Q}$

In the three cases, the parameters of the final path are shown in Table.4. It can be seen that after the dynamic 
path planning, the comprehensive detection probability is significantly reduced, but the range has increased. Compared with the original path, the cost value of the two paths with $\mathrm{M}$ and $\mathrm{N}$ as the start points is obviously reduced. For the case of point $\mathrm{Q}$ as start point, the dynamic planning is too late and the aircraft is too close to the new radar, although the dynamic planning makes the comprehensive detection probability is reduced, but the range increases, resulting in the cost is almost equal to the original path's.

Table 4. The parameters of different paths

\begin{tabular}{cccc}
\hline $\begin{array}{c}\text { Start } \\
\text { point }\end{array}$ & $\begin{array}{c}\text { Range } \\
(\mathbf{k m})\end{array}$ & $\begin{array}{c}\text { Comprehensive detection } \\
\text { probability }\end{array}$ & Cost \\
\hline $\begin{array}{c}\text { Original } \\
\text { path }\end{array}$ & 455.5 & 0.716 & 0.425 \\
M & 461.7 & 0.143 & 0.215 \\
N & 473.6 & 0.242 & 0.293 \\
Q & 472.2 & 0.593 & 0.429 \\
\hline
\end{tabular}

The simulation results show that the aircraft should observe the battlefield situation at any time and detect the new threat as soon as possible. After determining the enemy's new threat, the dynamic route planning should be carried out as soon as possible and integrated with the original optimization track.

\section{Conclusion}

A gradational algorithm is established that includes two layers of optimization algorithm. the first layer is the global path optimization completed by genetic algorithm using its global optimal performance. the second layer is a dynamic path optimization completed by $A^{*}$ algorithm using its dynamic characteristics. In flight, the aircraft flies along the global optimal path planed by the genetic algorithm. When a new threats was detected and its performance was determined, the $\mathrm{A}^{*}$ algorithm was used to give a local optimal path. Two paths was linked to combining the gradational path planning algorithm .. The range of the new optimal path would be longer than the original path, but the comprehensive detection probability is much lower than the original path. Considering the range and comprehensive detection probability, the cost is lower than the original path if taking the dynamic path planning before detected by the new threat.

\section{References}

1. HE Pei. Research on the Method of Low-altitude Penetration Track Planning. Beihang University, Beijing, China, 2003.

2. ZHENG Changwen, YAN Ping, DING Mingyue. Route Planning For Air Vehicles. National Defense Industry Press, Beijing, 2008.

3. WANG Jun, ZHOU Shudao, ZHU Guotao. Research of Common Route Planning Algorithms for Unmanned Air Vehicle. Fire Control \& Command Control, Vol. 37, No. 8 (2012), p. 5-9.

4. DU Ping, YANG Chun. Introduction of Air Vehicle Path Planning Algorithms. FLIGHT DYNAMICS, Vol. 23, No. 2 (2005), p. 10-14.

5. ZHANG Kao, ZHANG Yunfei, MA Dongli. Calculation and Analysis of Radar Stealth Performance of Aircraft. National Defense Industry Press, Beijing, 1997.

6. LIN Cuoyun, DONG Jiali. Method and Theory of Multi-objects Optimization. JiLin Education Press, Changchun, China, 1992.

7. LI Chunhua, ZHENG Changwen, ZHOU Chengping. Fast search algorithm for 3D-route planning. Journal of Astronautics, Vol. 23, No. 3 (2002), p. 13-17.

8. FAN Hongda, MA Xiangling, YE Wen. Low Altitude Penetration Route Planning Technology for Aircraft. National Defense Industry Press, Beijing, 2008.

9. LI Xia, XIE Jun, et al. Path Panning for UAV Based on Improved Heuristic A* Algorithm. The Ninth International Conference on Electronic Measurement \& Instruments(3) (2009), p.488-493

10. HE Wang, QING Li, and NONG Cheng. Real-time Path Planning for Low Altitude Flight Based on A* Algorithm and TF/TA Algorithm*. $8^{\text {th }}$ IEEE International Conference on Automation Science and Engineering(2012), p. 837-842 